sources. In otherwords, Wilson calls for treating biodiversity as a natural resource with real economic value, like rivers or forests. If a particular fungi turns out to be the source of an anticholesterol drug, as it did for Merck (Rahway, NJ), that fungi should have an economic value and be protected. This has very concrete meaning for the biotechnology industry and biologists alike. The development costs of millions of dollars to find such organisms may be well worth it. Merck's fungi-derived anticholesterol drug Mevacor, for example, posted sales of $\$ 750$ billion dollars in 1990 .

In short, the entire premise of Wilson's strategy for preserving biodiversity is a highly utilitarian concept that puts a price on genetic diversity and accepts the price of saving some habitats, inevitably, at the expense of others. Some will be more important biologically, others will pay their way, still others may pass on to developers. Nowhere in the Science article is there any inkling that the authors of the Wildlands Project considered the economic value of the habitats they propose setting aside. Nowhere is there any indication that there was any attempt to calibrate the plan in terms of the actual contribution North America makes to the global genetic library that Wilson and others conservation biologists continually invoke, perhaps because they know that, for the most part, it is of comparatively little value.

Nowhere is there any hint that protecting the genetic diversity of the planet has little to do with giving grizzlies, mountain lions, and wolves the room to roam free in a state, as one of the plan's authors put it, of "fierceness." Rather, as Wilson and Ehrlich know, it has to do with laborious cataloguing of the 85 percent of the species out there that we still know nothing about. From a strictly political perspective, it does not help that the sexiest of these creatures are plants, worms, mites, nematodes, insects, and microorganisms, which may represent a healthy slice of the future for the biotechnology industry. In biodiversity, as in life, it helps to know who your allies are, and just why it is they're your allies. I/I

\title{
Impacting biological weapons
}

WASHINGTON, D.C.-Biotechnology both complicates and simplifies enforcement of the international Biological Weapons (BW) Convention, which the U.S. signed in 1972. On the complicating side, biotechnology provides plenty of new opportunities for ambiguous undertakings, often called "dual use" because seemingly innocuous ventures might be cloaking forbidden BW-making activities. But, on the simplifying side, biotechnology offers exquisitely sensitive methods for detecting and identifying pathogens and other potential BW agents. Such methods could eventually provide more effective ways to expose illicit activities, thereby enforcing the BW Convention.

Recently, representatives from the Biotechnology Industry Organization (BIO, Washington, DC) and the Pharmaceutical Manufacturers Association (PMA, Washington, DC) completed a report outlining industry qualms over proposed BWrelated disclosure requirements. "We have a grave concern that the imposition of intrusive verification measures involving multilateral inspections could result in the potential loss of key technologies to other countries," the report says.

Two kinds of inspection visits are envisioned, those that are "routine and scheduled" and those, called "challenge visits," that are prompted by suspicions of BW activity and would be more intrusive. Industry representatives are concerned that such visits could be abused for the purposes of industrial espionage. However, they acknowledge that other kinds of inspections on industry sites take place already without apparent abuse, including those required by federal regulatory agencies. "These agencies have a history of not sharing proprietary information with competitors," the BIO-PMA report notes.

Many industry concerns can be satisfactorily addressed, argue representatives from the Federation of American Scientists (FAS, Washington, DC), who point out that the inspections now being proposed to assure BW compliance are "less stringent, particularly with respect to the auditing of records, than those of the Food and Drug Administra- tion and similar agencies in other countries."

At the international level, negotiators have identified a set of 21 measures, including satellite surveillance and on-site monitoring and inspections, to help assure that the $\mathrm{BW}$ Convention is not being violated. Most observers agree that these measures fall short of offering verification of compliance. Instead, they represent steps that are "confidence building" in a situation where verification in the literal sense of the word appears impossible.

For their part, advances on the technological front could help certify that BW violations are not occurring. Some of these advances are coming from researchers within the Department of Defense (DoD, Washington, DC) who are developing rugged and highly sensitive tests for detecting and identifying potential BW agents. Although designed for identifying BW agents being deployed in battlefield settings, the tests under development conceivably could provide a means for detecting activities that violate the BW Convention.

Researchers at the Naval Medical Research Institute (Bethesda, MD) are detecting PCR-amplified DNA products with a highly sensitive biosensor as a means of identifying suspected pathogens. "For each agent, we have a set of primers and two sets of specific probes to give a high level of specificity and no ambiguity," says the institute's Mitchell Carl. The pathogen detection system is also highly sensitive. For example, as few as six spores of Bacillus anthracis, abacterial pathogen that might be deployed as a BW agent, can be detected within a few hours time.

Applications extend beyond the battlefield, as the detection system should be generally useful for monitoring pathogens in settings where standard diagnostic techniques either are too slow or too cumbersome. The system "should work with any viral or bacterial pathogen as long as the DNA can be extracted for a battery of indicator tests,"Carl says. Currently, the group has developed probes for about a halfdozen potential BW agents.

-Jeffrey L. Fox
Biotech allows

seemingly

\section{innocuous}

ventures to cloak

forbidden

biological-

weapon (BW)

activities. But it

also offers new

ways to detect

BW agents. 